\title{
Theory of 3F4D Universe (Beyond Standard Model of Particle Physics)
}

\author{
Yogesh Vishwanath Chavan \\ Independent Researcher \\ Email: omsai_333@rediffmail.com
}

How to cite this paper: Chavan, Y.V. (2021) Theory of 3F4D Universe (Beyond Standard Model of Particle Physics). Open Journal of Microphysics, 11, 9-26. https://doi.org/10.4236/ojm.2021.112002

Received: May 6, 2021

Accepted: May 27, 2021

Published: May 30, 2021

Copyright $\odot 2021$ by author(s) and Scientific Research Publishing Inc. This work is licensed under the Creative Commons Attribution International License (CC BY 4.0).

http://creativecommons.org/licenses/by/4.0/ (c) (i) Open Access

\begin{abstract}
In this theory, "mass is equivalent to length of imaginary straight line segment" and "direction to imaginary line segment is intrinsic property of that particle which is Equivalent to its intrinsic spin". With this concept, all fundamental particles, fermions and bosons are described as quanta imaginary string particles with a definite direction. For e.g. unidirectional imaginary straight line with fixed length are massive spin 1/2 fermions; while unidirectional imaginary quanta curved lines are massless spin 1 bosons. Thus, it gives co-relation between massless (curved imaginary line) and spin $=1$ properties of boson as proved in QED theory. All fundamental particles of standard model and beyond standard model are arranged in one simple diagram in 3 folds (bottom fold, middle upper and lower folds and top fold) and are projected in $4^{\text {th }}$ Imaginary Dimension in order of decreased in Mass from $\mathrm{TeV}$ to approx. $0 \mathrm{eV}$. This theory is beyond standard model because it predicts new fundamental particles viz. dark matter ( $\operatorname{spin}=0$ massive boson) along with gravitons ( spin $=2$, massless bosons); $4^{\text {th }}$ pair of neutrinos, vertical massless boson $(\mathrm{VMB})$ particles and tri-axis massive boson $($ spin $=0)$ particle. The discovery of these new particles will act as solid proof to this theory. With this $3 \mathrm{~F} 4 \mathrm{D}$ representation of the universe at an atomic and sub-atomic level, it solves lots of current problems of SM of particle physics like matter-antimatter asymmetry, origin of mass of hadrons like protons, origin of mass and L.H. nature for neutrinos, wave-particle duality of particles, etc. giving true insight of fundamental particles. With proving that, dark matter is not a quanta particle, rather it is a single entity and spreads/expands throughout the universe in the form of "web of spider", it shows space-time is not empty, but it is filled with Continuous lines of Dark Matter and we, materialistic massive objects are floating/sailing w.r.t. current of this vast ocean. Correlation of its continuity with time gives new a definition to time: "Time is neither Illusion nor $4^{\text {th }}$ Dimension, but, it represents Continuous Flow of Single Entity, Dark Matter". Space-time is not Empty and is filled with mas-
\end{abstract}


sive dark matter, hence, we have to add more terms to newtonian gravitational equation to account for gravitational strength of mass of surrounding dark matter which represents curvature of space-time in terms of Increase in its mass-density w.r.t. to mass-density of flat universe. Finally, using an empirical formula $\left(h=k^{\star} c^{\star} \mathrm{Q} ; \mathrm{k}=\right.$ boltzmann's constant $=$ mass in $\mathrm{TeV}$ range $)$ and inverse relation of mass-density of DM with age of the universe $\left(\mathrm{H}^{2}=\right.$ Constant.G.Mass-Density of DM); Unification of Gravity at $\mathrm{TeV}$ is achieved without consideration of "Gravitational Constant, G", hence, Planck's Scale is not required.

\section{Keywords}

Unification, Particle Physics, Standard Model, Dark Matter, Gravitons

\section{Introduction (The Standard Model of Particle Physics) [1]}

In the 17th century, scientists came to the conclusion that chemical elements such as hydrogen, oxygen, and copper were composed of atoms. A chemical element, hydrogen say, was thought to consist of a single type of atom. And chemical methods made it possible to determine the approximate size of such an atom: $10^{-8} \mathrm{~cm}$ that means one billion hydrogen atoms stacked on top of one another would reach a height of around $10 \mathrm{~cm}$ (See Figure 1).

Today, we know that normal matter is made up of quarks (which are the building blocks of the atomic nucleus) and electrons. The standard model describes the fundamental interactions in a simple form. The interactions are the chromo dynamic force (QCD), which acts between quarks and gluons, and the electroweak force, which acts between $\mathrm{W} \pm, \mathrm{Z}_{0}$ bosons and quarks, charged leptons, such as electrons. Today's standard model of elementary particles, which consists of the theory of the electroweak interaction plus QCD, seems to describe the whole of physics (See Table 1).

In the standard model, leptons and quarks are points, singularities in space. Can such infinitely small points even have a mass? In order to introduce leptons and quarks masses, physicists invented a hypothetical field which is able to give mass to these particles. The field is called the Higgs field (

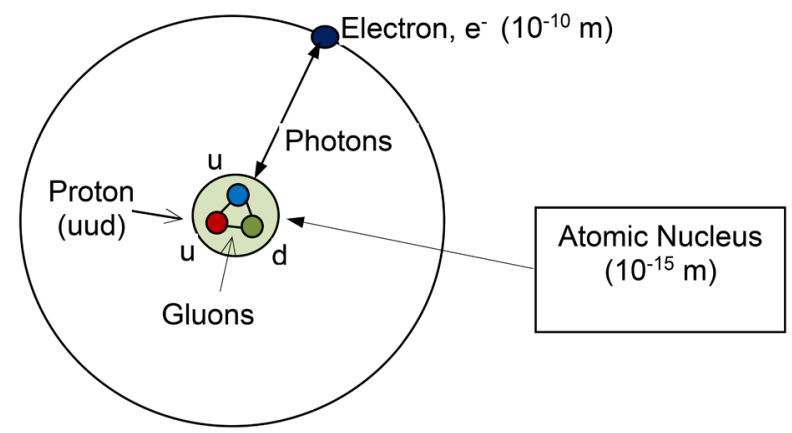

Figure 1. Hydrogen atom. 
Table 1. The standard model of particle physics [2].

\begin{tabular}{|c|c|c|c|c|}
\hline \multicolumn{5}{|c|}{ The Standard Model } \\
\hline \multicolumn{5}{|c|}{ (Matter \& Energy; $\left.\mathrm{E}=\mathrm{m}^{\star} \mathrm{c}^{2}\right)$} \\
\hline Forces \& & Boson Particles & & Fermion Particles (Spin $= \pm 1 / 2)$ & \\
\hline $\begin{array}{l}\text { Strong Nuclear } \\
\text { Force (SNF); }\end{array}$ & $\begin{array}{l}\text { Gluon }(\text { Spin }=1) \\
\quad \text { Charge }=0 \\
\text { Massless Particle }\end{array}$ & $\begin{array}{l}3 \text { Generations } \\
\mathbf{u} \\
\text { (Up Quark) Charge }=+2 / 3 \mathrm{Q} \\
\text { Mass }=1-5 \mathrm{MeV}\end{array}$ & $\begin{array}{c}\text { of Quarks (Always confined insic } \\
\text { c } \\
\text { (Charm Quark) Charge }=+2 / 3 \mathrm{Q} \\
\text { Mass }=1-1.3 \mathrm{GeV}\end{array}$ & $\begin{array}{l}\text { de Hadrons) } \\
\qquad \begin{array}{c}\mathbf{t} \\
\text { (Top Quark) Charge }=+2 / 3 \mathrm{Q} \\
\text { Mass }=174.3 \mathrm{GeV}\end{array}\end{array}$ \\
\hline $\begin{array}{l}\text { Electromagnetic } \\
\text { Force (EMF) }\end{array}$ & $\begin{array}{l}\text { Photon }(\text { Spin }=1) \\
\text { Charge }=0 \\
\text { Massless Particle }\end{array}$ & $\begin{array}{c}\mathrm{d} \\
\text { (Down Quark) } \text { Charge }=-1 / 3 \mathrm{Q} \\
\text { Mass }=3-9 \mathrm{MeV}\end{array}$ & $\begin{array}{c}\text { s } \\
\text { (Strange Quark) Charge }=-1 / 3 \mathrm{Q} \\
\text { Mass }=70-170 \mathrm{MeV}\end{array}$ & $\begin{array}{c}\text { b } \\
\begin{array}{c}\text { (Bottom Quark) Charge } \\
=-1 / 3 \mathrm{Q} \\
\text { Mass }=4-4.4 \mathrm{GeV}\end{array}\end{array}$ \\
\hline $\begin{array}{l}\text { Weak Nuclear } \\
\text { Force (WNF) }\end{array}$ & $\begin{array}{c}\mathrm{W}^{+}, \mathrm{W}^{-}, \mathrm{Z} \\
(3 \text { Boson Particles) } \\
(\text { Spin }=1) \\
\text { Mass of } \mathrm{W}^{+/-}=80 \mathrm{GeV} ; \\
\text { Mass of } \mathrm{Z}=91 \mathrm{GeV}\end{array}$ & $\begin{array}{c}\mathrm{e}^{-} \\
\text {(Electron) } \\
\text { Charge }=-\mathrm{Q} \\
\text { Mass }=0.51 \mathrm{MeV}\end{array}$ & $\begin{array}{c}3 \text { Generations of Leptons } \\
\mu^{-} \\
(\text {Muon }) \\
\text { Charge }=-\mathrm{Q} \\
\text { Mass }=105.6 \mathrm{MeV}\end{array}$ & $\begin{array}{c}\tau^{-} \\
(\mathrm{Tau}) \\
\text { Charge }=-\mathrm{Q} \\
\text { Mass }=1.78 \mathrm{GeV}\end{array}$ \\
\hline $\begin{array}{c}\text { Gravitational } \\
\text { Force (GF) }\end{array}$ & $\begin{array}{l}\text { Graviton }(\text { Spin }=2) \\
\text { Charge }=0 \\
\text { Massless Particle? }\end{array}$ & $\begin{array}{c}v_{\mathbf{e}} \\
\text { (Electron Neutrino) } \\
\text { Charge }=0 \\
\text { Massless Particle? }\end{array}$ & $\begin{array}{c}\boldsymbol{v}_{\mu} \\
\text { (Muon Neutrino) } \\
\text { Charge }=0 \\
\text { Massless Particle? }\end{array}$ & $\begin{array}{c}\boldsymbol{\nu}_{\boldsymbol{\tau}} \\
\text { (Tau Neutrino) } \\
\text { Charge }=0 \\
\text { Massless Particle? }\end{array}$ \\
\hline
\end{tabular}

(For every Fermion; there exists its Anti-Particle).

theoretician Peter Higgs, who introduced this field [3] Ch. 11.

On July 4, 2012, the Large Hadron Collider at CERN announced results consistent with the massive higgs particle (God particle, spin $=0$ ). Could this theory prove to be a last and thereby final truth? There are a number of important questions not answered by this theory, like constant mass of particles (fermions), matter-antimatter asymmetry, nature of particles related to $23 \%$ gravitating dark matter \& around 73\% repulsive dark energy [4] Ch. 44, Union of Einstein's Gravity Force (manifestation of the curvature of space and time; continuous nature of space-time) with physical forces (particle concept; quanta nature) like EMF, SNF \& WNF of Standard Model, origin of 3 generations or families of Fermions viz. Quarks \& Leptons, etc.

\section{Three Postulates and Equivalence of Mass with Imaginary Straight Line [6]}

In this theory, instead of considering grand unification of forces at around Planck's scale i.e. $10^{18} \mathrm{GeV}$; it is showed that the Universe itself came into existence by "Unified massive fundamental particle" at TeV Range. The theory is based upon three postulates, viz.

1) Current experiments limit on size of leptons and quarks up to $10^{-19} \mathrm{~m}$.

2) "Empirical formula" is derived by myself. It connects 4 main fundamental constants of nuclear physics as well as satisfies above first experimental aspect. 


$$
\mathrm{h}=\mathrm{k}^{\star} \mathrm{c}^{\star} \mathrm{Q} \Leftrightarrow \mathrm{m}^{\star} \mathrm{V}^{\star} \lambda=\mathrm{m}^{\star} \mathrm{V}^{\star}\left(2 \Pi^{\star} \mathrm{r}\right) \text { Where; }\{\mathrm{k} /(2 \Pi)\} \Leftrightarrow \mathrm{m}, \mathrm{c} \Leftrightarrow \mathrm{V} \text { and } \mathrm{Q} \Leftrightarrow
$$

r

$\mathrm{h}=$ Planck's constant $=6.626 \times 10^{-34} \mathrm{~J} . \mathrm{Sec}$

$\mathrm{k}=$ Boltzmann's Constant $=1.38 \times 10^{-23} \mathrm{Kg} \&\{\mathrm{k} /(2 \Pi)\}=$ Maximum Mass of Particle $=1.23 \mathrm{TeV}$

$\mathrm{c}=$ Speed of Light $=2.997 \times 10^{8} \mathrm{~m} / \mathrm{Sec}$.

$\mathrm{Q}=$ Charge on Particle $=$ Minimum Quanta Size of Particle $=1.602 \times 10^{-19} \mathrm{~m}$.

The important thing about this formula is that for given experimental limit on minimum size of basic particles (which is equal to value of charge $Q$ ) and for limit on maximum speed of particle viz. speed of light, c; it gives limit on maximum mass of particle $(\mathrm{k})$ in the range of $\mathrm{TeV}$. This formula tells us that the universe must had started from above "unified massive fundamental particle" by uniting above constants viz. h, k, c \& Q. creation of the universe at $\mathrm{TeV}$ range means no need of Planck's scale. Planck's scale depends upon gravitational constant " $G$ " and this scale is required to unify Gravity which is very weak as compared to other forces. At this scale, gravity which depends upon value " $G$ ", becomes as strong as other 3 atomic and sub atomic fundamental forces. It means, to achieve Unification of Gravity at $\mathrm{TeV}$ range, we should not consider Gravitational constant "G" in calculating gravitational coupling strength, but there must be another way to define Gravity which we will see later.

3) Equivalence of mass and imaginary line: in string theory, particles are defined in string like manner (small one dimensional object) in real space with size equal to Planck length around $10^{-33} \mathrm{~cm}$ in order to avoid singularity which happens in case of point like object. However, we have to accept 11 dimensions instead of our 4 dimensional space-time.

But, when I attempted to define, "one dimensional line"; I came to realize that, for existence of such line in real space, its thickness adds at least one more dimension in real space. Then, this thickness, itself spoils the definition of "one dimensional line" in real space. Line with zero thickness is meaningless from point of view of real space (i.e. Line doesn't exist in real space). In other words, "It is impossible to draw one dimensional Line in real space" as shown in Figure 2.

While above thing doesn't happen in case of "imaginary space". Thickness of line (imaginary line) doesn't spoil the definition of "one dimensional line" in imaginary space. This is because one can't measure imaginary thickness in terms of fixed scale like $\mathrm{cm}$ or $\mathrm{m}$.

Now, from physics point of view, to draw any Line, you will require some Entity i.e. Amount of Matter or Mass. Then, from above math's point of view, mass should embed on imaginary line itself. Thus,

"Mass is equivalent to length of straight imaginary line"

Keeping in mind of above 3 postulates and tripartite nature of particles (3 generations or families of fermions), in this theory, the particles of standard model are arranged as per their quantum properties in 3 folds way (bottom fold, 


\section{$3^{\text {RD }}$ POSTULATE OF THE THEORY}

\section{Equivalence of Mass and Imaginary Line:}

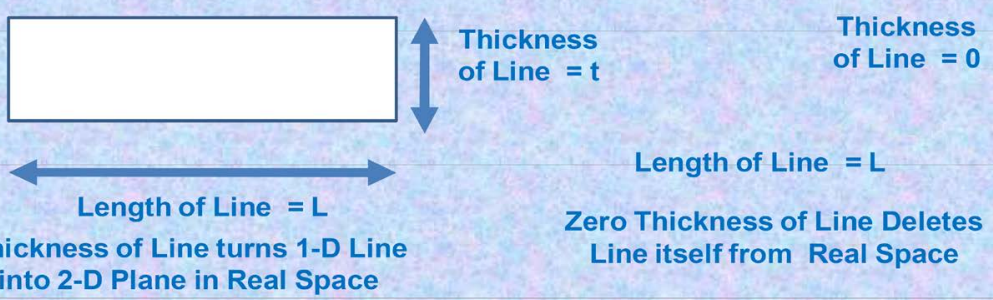

\section{In Real Space,}

\section{One Dimensional Line doesn't Exists}

Figure 2. Nature of one dimensional line in real space.

middle folds $\&$ top fold) from $\mathrm{TeV}$ to approx. $0 \mathrm{eV}$ in $4^{\text {th }}$ Imaginary Dimension (Imaginary Line $\equiv$ Mass) i.e. instead of point like structure, all fermions act like one dimensional straight imaginary lines. These particles have no thickness, hence from our 3 dimensional real space, we always look them as point like or structure less particles. "Direction to imaginary line is intrinsic property of that particle and is equivalent to spin". Thus, one dimensional unidirectional straight line is equivalent to intrinsic angular momentum of that particle called as $1 / 2$ spin; while particle with curved (means more than one dimensional) unidirectional line will have spin equal to 1 called as boson particles. Therefore, these force carrying particles, Bosons are massless particles (mass means straight imaginary Line). With this representation, massless property of boson is directly linked with its spin $=1$ property which is already proved in QED theory. Also, massless particles are quanta (i.e. particle) curved (i.e. wave) imaginary lines giving wave-particle duality nature to them [5] Ch. 9, as proved in quantum mechanics. One interesting point here, I want to say that you are not seeing straight or curved imaginary lines; but they are actually Massive Fermions and massless bosons respectively and you are seeing them by your naked eyes (see Figure 3).

Note: Imaginary Straight Lines with downward direction in 4th Imaginary Dimension are Anti Fermions.

\section{Creation of 3 Folds and Fundamental Particles in $4^{\text {th }}$ Imaginary Dimension [6]}

Based upon above 3rd postulate and tripartite nature of particles, they are arranged in 4th Imaginary dimension in different folds as per their by masses and other quantum properties like electrical charge, spin etc. (see Figure 4). Straight 


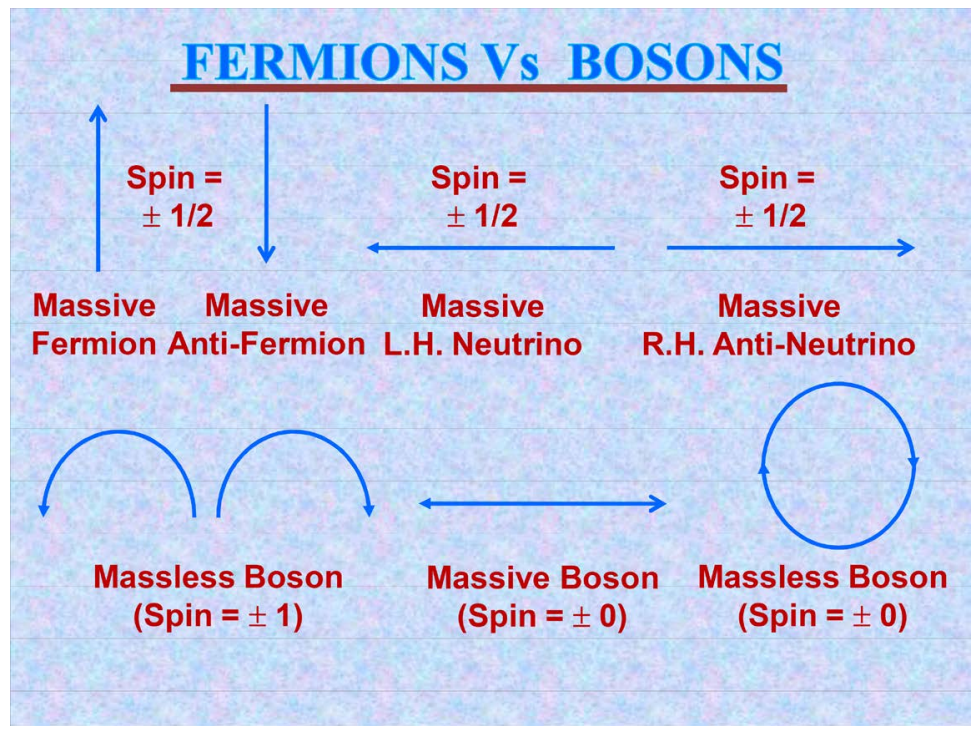

Figure 3. Massive fermions vs massless bosons.

\section{Folds 4-D Universe}

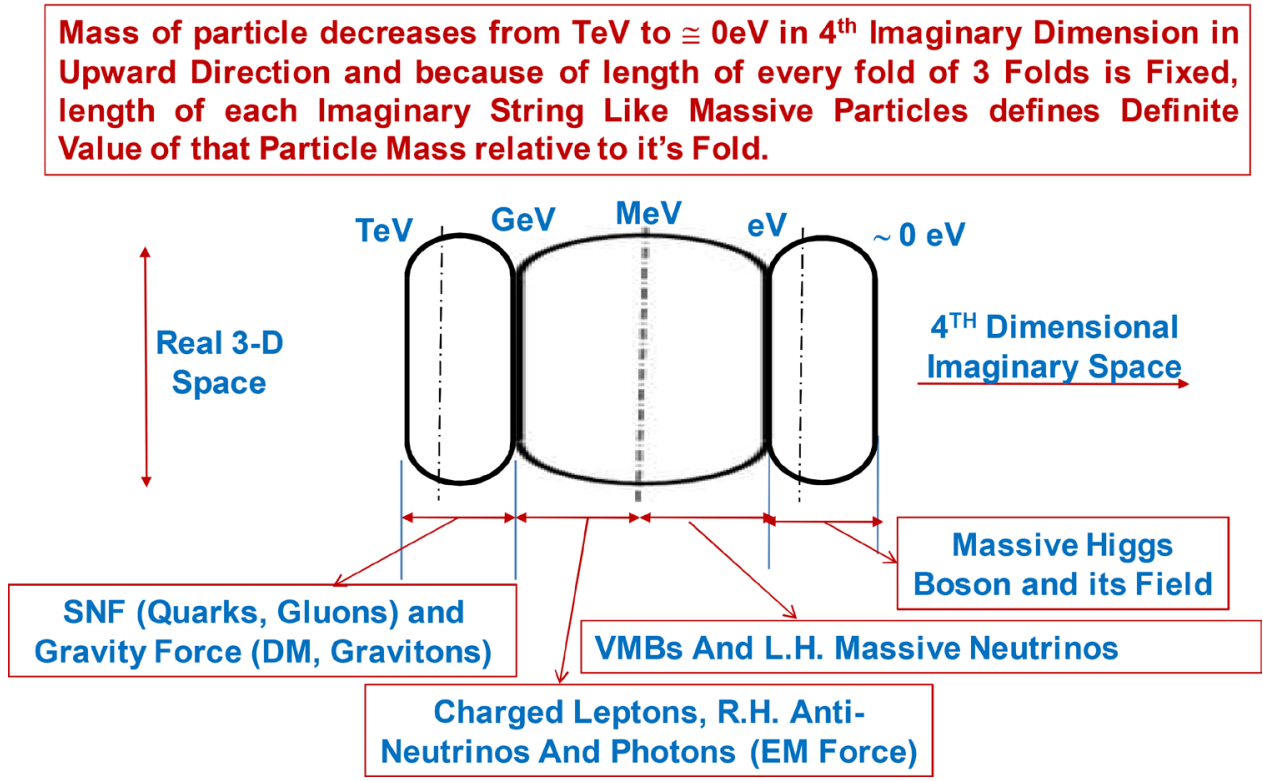

Figure 4. Arrangement of fundamental particles ( 3 folded way) in $4^{\text {th }}$ imaginary dimension.

Imaginary line acts like massive particle with definite value of mass relative to its fold. Unfolding of universe started from bottom fold, then top fold and step by step middle upper and lower folds between them. Let us see each fold and particles within them.

In Figure 5, you will clearly see how particles are created through step by step unfolding of folds/layers i.e. creation of top fold from bottom fold and later middle folds between them. Thus, all particles are required and they are definitely ordered by God to create the universe in beautiful and elegant way. 


\section{Description of 3 Folds and Nature of Particles}

\subsection{Bottom Fold}

As shown in Figure 6, the creation of the universe was started with "bottom fold". It is vertically divided into 2 sub-folds in ratio of $2: 1$ w.r.t. zero reference line as shown in Figure 6 and each sub fold carries Fractional Charged

\section{Creation of Universe in 3 Folds Way into $4^{\text {th }}$ Dimension (Imaginary Space)}

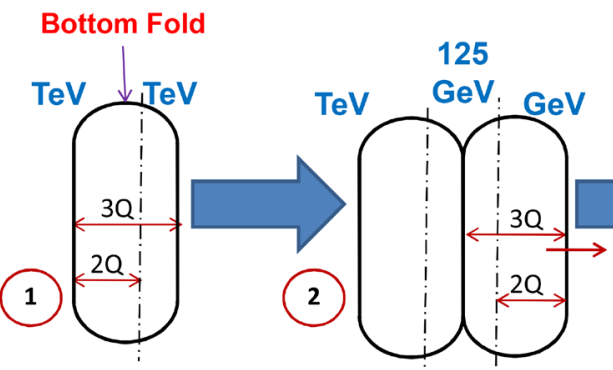

(3)
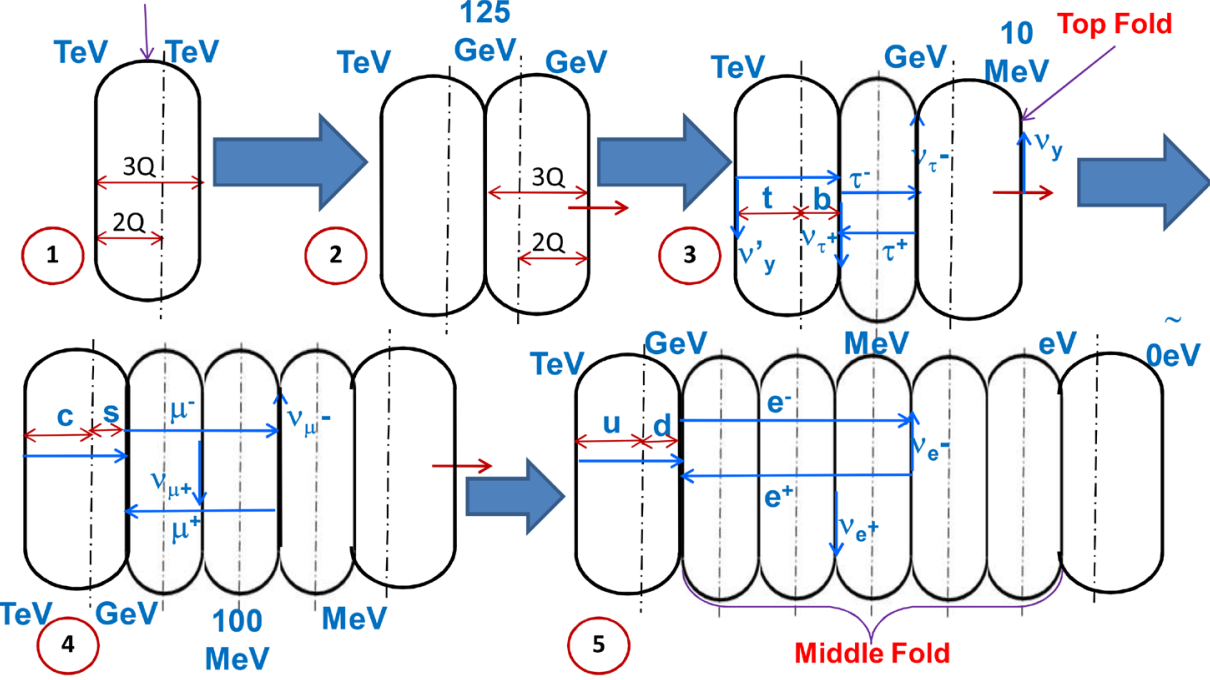

\{Note: Actual Bottom Fold Size is $3 Q$; out of which top quarks shared only $2 Q$ size i.e. $2 / 3^{\text {rd }}$ part of Bottom Fold. Hence, Charge on top quarks is (+2/3). Same thing is applied to Bottom Quark with Minus Sign on Charge (i.e. -1/3).\}

Figure 5. Creation of 3 folds and fundamental particles in $4^{\text {th }}$ imaginary dimension.

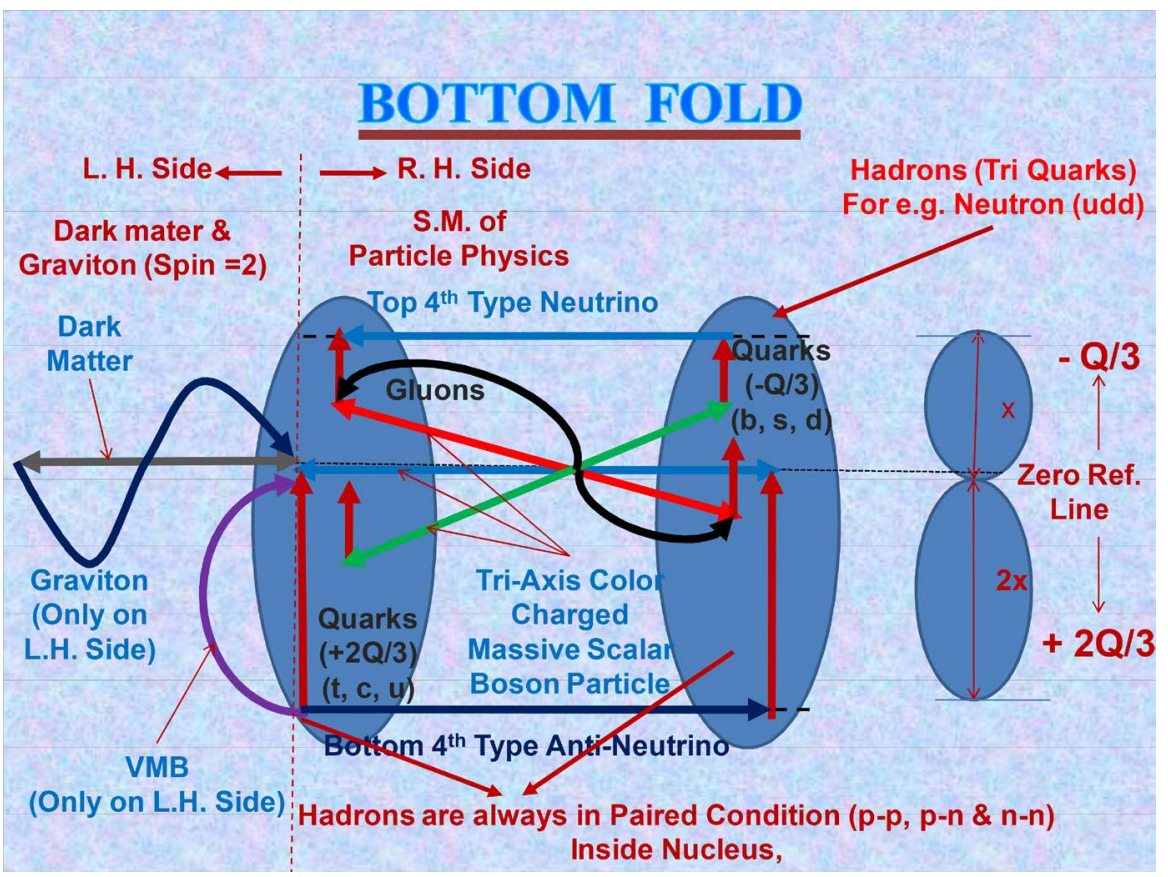

Figure 6. Bottom fold. 
particles $(+2 / 3 \mathrm{Q} \&-1 / 3 \mathrm{Q}$ respectively) called as quarks. Same $(+2 / 3 \mathrm{Q})$ bottom sub-fold of bottom fold is shared by top, charm \& up quarks; while same $(-1 / 3$ Q) top sub-fold of bottom fold is shared by bottom, strange \& down quarks. Hence, mass of quarks is like mass parameter type. "Quantization of charge" is correlated with quanta height of folds and its sub-folds.

This bottom fold is partitioned horizontally into 2 parts. R.H. part contains particles related to hadrons viz. 6 types of quarks, gluons and new predicted particles like 4th pair of neutrinos, L.H. vertical mass less boson (VMB) particle and tri-axis massive boson $($ spin $=0)$ particle (trident) which gives mass to hadrons. Discovery of these particles will act as solid proof to this theory. This massive trident particle is made up of 3 massive boson ( which are arranged in 3-D space in the form of tri-axis and are responsible for color charge property related to 3 quarks. Red, green, blue color charges are represented by $\mathrm{X}, \mathrm{Y}$ and $\mathrm{Z}$ arms of trident particle (L.H. side); while anti-red, anti-green, anti-blue color charges are denoted by X', Y' and Z' arms (R.H. Side). From above Figure 6, it is cleared that, baryons like protons and neutrons inside atomic nucleus should always remain in paired form i.e. $\mathrm{p}-\mathrm{p}, \mathrm{p}-\mathrm{n}$ or $\mathrm{n}-\mathrm{n}$. Quarks are at boundary of atomic nucleus and these quarks prevent gluons to escape from atomic nucleus. It is not the confinement of quarks, but it is confinement of gluons which is required for formation of atomic nucleus. Without this wall of quarks, formation of atomic nucleus (i.e. materialistic object) is impossible. Our universe will be like dark soup of trident-gluon plasma. Gluons can't escape in bottom fold into 3-D space; but, in certain conditions, gluons can escape into $4^{\text {th }}$ imaginary Dimension in the form of massless photons at lower middle fold.

During beginning of the universe, VMB particle was connected with only L.H. anti-quarks (quarks were on R.H. side and anti-quarks were on L.H. side.) as shown in Figure 6 and has major role in conversion of anti-quarks into Quarks through expansion of the Universe in 4th imaginary dimension by creation of top fold above bottom fold. This conversion was happened as shown in Figure 7: release of pressure of 4th type of top neutrino on anti-quark by vertical upward rotational movement of L.H. side VMB particles. This vertical rotational upward movement not only released pressure of top neutrino, but it simultaneously converted anti-quarks into quarks through its internal rotation with no violation of law of charge conservation i.e. total charge of all 3 anti-quarks (anti-baryon like anti-neutron) before conversion remains same equal to their total charge after conversion into quarks and is equal to zero. Thus, this theory though new VMB particle, gives "ingenious solution to matter-antimatter asymmetry problem".

L.H. side of bottom fold consist of massive boson ( "dark matter" surrounded by massless boson (spin $=2$ ) graviton (carrier of gravity force). Graviton is made up of 2 Spin $=1$ massless bosons, hence, it has Spin $=2$. Graviton connects with dark matter as well as with baryons (materialistic 


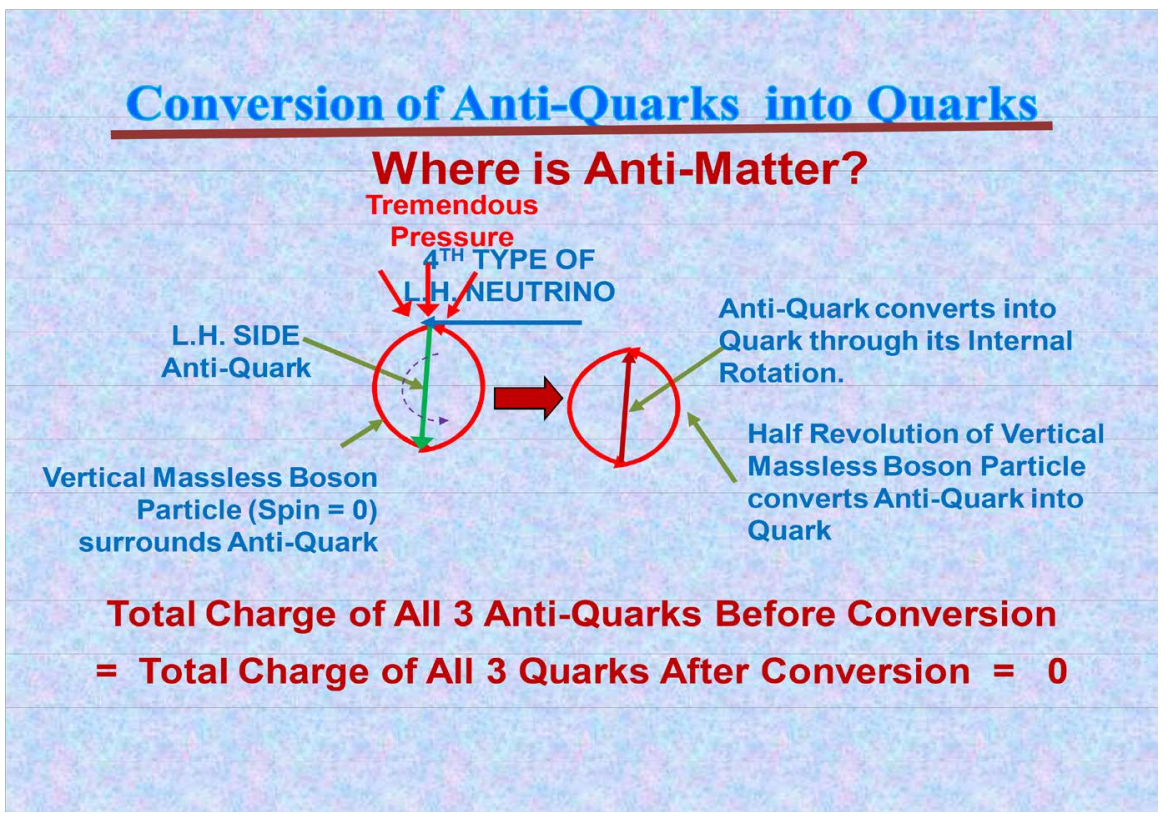

Figure 7. Conversion of anti-quarks (anti-matter) into quarks (matter).

objects like stars, planets etc.) and has direction towards baryons (atomic nucleus). Thus, gravity force should be attractive in nature. Also, as we know, Mass of materialistic objects is entirely due to mass of atomic nucleus, hence, connection of gravitons only with atomic nucleus (hadrons) i.e. at bottom fold clearly shows that materialistic objects gravitate because of their mass as per gravitational equation and it does not depend upon size of the object. It means gravitons never interact with mass of charged leptons (e.g. electrons) or charge less leptons (e.g. neutrinos) or massless photons, because they are at middle folds.

R.H. side contains fundamental particles as described by standard model. existence of dark matter and gravitons at L.H. side shows that these particles are beyond the scope of SM of particle physics. This is the main reason, why dark matter not radiates or interacts with other Fundamental particles. As there is no restriction to dark matter (no confinement like quarks confinement), it will spread away from atomic nucleus along with gravitons making expansion of the universe. Thus, dark matter no longer remains as quanta particle, but by connecting to each other, it acts like single entity making giant network structure like "web of spider". These continuous lines of dark matter with gravitons form continuous space-time as shown in Figure 8 which is essential part of Einstein's general theory of relativity. That is, space-time is neither empty nor have quanta aspect, but these continuous lines of dark matter themselves represents continuous space-time. Mass-density of dark matter decreases with expansion i.e. increase in age of the universe $\left.\left\{\mathrm{H}^{2}=\left(8^{\star} \mathrm{Pi} / 3\right)^{\star} \mathrm{G}^{\star} \text { (Mass-Density }\right)_{\mathrm{DM}}\right\}$. Compression of space-time means increase in density of dark matter. With this notion, we can convert, Einstein's concept of Curvature of Space-time as gravity effect into increase in mass-density of dark matter (compressed dark matter) w.r.t. mass-density of flat universe. Increased mass-density of dark matter means less 


\section{Dark Matter And Graviton (In Bottom Fold)}

Gravitons spreads away from Center of Matter (Nucleus at Bottom Fold) along the imaginary Web Line of Dark Matter, which in the form of Single Entity makes Continuity in Space-Time.

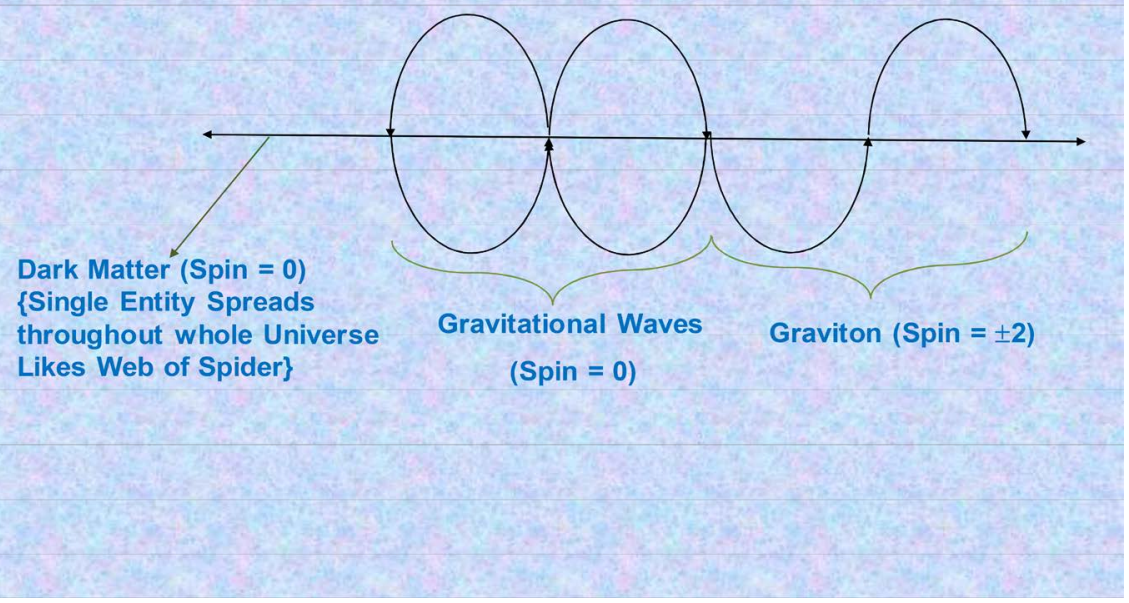

Figure 8. Dark matter (single entity like web of spider) and gravitational waves (Spin = $0)$.

time (i.e. decrease in age of the universe in that region) as compared to age of the universe of flat universe. In other words, mass-density of surrounding dark matter near to massive object is more (compressed dark matter due to gravitational pull of that massive object) as compare to mass-density of dark matter of flat universe and it is called as time dilation effect due to gravity. From, this relation between space-time and mass-density of dark matter, we have true definition of time:

"Time is neither illusion nor $4^{\text {th }}$ dimension, but, it represents continuous flow of single entity, dark matter".

Mass-density of flat universe is equal to absolute time and we all experience this absolute time at same moment everywhere, because of continuous expanding lines of single entity, dark matter. conversion of Einstein's GR concept of gravity, as curved space-time into increase in mass-density of massive DM; adds more terms into equation of gravitational strength (g) apart from Newtonian Gravitational Strength $\left(G^{\star} M / R^{2}\right)$ to account for gravitational effect of mass of surrounding DM around that massive object. Thus, $1^{\text {st }}$ Term is usual Newtonian Term $\left(\mathrm{g} 1=\mathrm{G}^{*} \mathrm{M} / \mathrm{R}^{2}\right), 2^{\text {nd }}$ Term $\left(\mathrm{g} 2=\mathrm{H}^{2 *} \mathrm{R}_{\mathrm{U}}\right)$ represents gravitational strength of flat universe, while $3^{\text {rd }}$ Term represents gravitational strength of mass of surrounding Massive dark matter $\left(\mathrm{M}_{\mathrm{DM}}\right)$ because of increase in its Mass-density [ $\mathrm{g} 3$ $\left.=\mathrm{G}^{*} \mathrm{M}_{\mathrm{DM}} / \mathrm{R}^{2}=\left\{\left(4^{*} \mathrm{Pi} / 3\right)^{*} \mathrm{G}^{*}\left(\rho_{\mathrm{DM}}\right)^{*} \mathrm{R}\right\}\right]$. In current universe (Age $=13.8$ Billion Years), Mass-density of dark matter $\left(\rho_{\mathrm{DM}}\right)$ is very less around $10^{-27} \mathrm{Kg} / \mathrm{m}^{3}$ $\left\{(\rho D M) \propto \mathrm{H}^{2}\right\}$; hence, $\mathrm{g} 1 \gg(\mathrm{g} 2+\mathrm{g} 3)$ and therefore, $(\mathrm{g} 2+\mathrm{g} 3)$ can be neglected for gravity effect of massive objects like the Earth, the Sun etc. But, for same massive object of mass, $\mathrm{M}$, (g1) over given fixed distance $\mathrm{R}$ always remains constant (Value of g1 will not change with change in age of the Universe); while 
with decrease in Age of the Universe, Mass-Density of Dark Matter increases and with this Dynamic Gravity effect of Dark Matter, value of $(\mathrm{g} 2+\mathrm{g} 3)$ may exceed value of g1. For e.g. during creation of the Universe, at quanta level i.e. for $\mathrm{M}=\mathrm{k}, \mathrm{R}=\mathrm{Q} \& \mathrm{H}=\mathrm{c} / \mathrm{Q} ;\left(\mathrm{g} 1=\mathrm{G}^{*} \mathrm{k} / \mathrm{Q}^{2} \cong 10^{4} \mathrm{~m} / \mathrm{Sec}^{2}\right)$ and $\left(\mathrm{g} 2=\mathrm{g} 3 \cong \mathrm{c}^{2} / \mathrm{Q}=10^{35}\right.$ $\left.\mathrm{m} / \mathrm{Sec}^{2}\right)$ i.e. $\{\mathrm{g} 1 \ll(\mathrm{g} 2+\mathrm{g} 3)\}$ It means, at quanta level, during creation of the Universe, role of “G” i.e. Newtonian Gravitational Term (Time Independent Gravity) becomes insignificant. In other words, for Unification of Gravity with other forces, we do not have to depend upon Planck's Scale which is based upon "G". But, through Dynamic Gravity of Dark Matter, calculations at TeV Range (Mass = Value of k; Boltzmann's Constant) shows that, Gravitational Coupling Strength becomes equal to "1" i.e. Ratio of these additional terms $\left(\mathrm{Q}^{2 *} \mathrm{c}^{*} \mathrm{k}\right)$ to $\left(\mathrm{h}^{*} \mathrm{c}\right)$ has value equal to "Unity"; $\left\{\left(\mathrm{Q}^{2 *} \mathrm{c}^{\star} \mathrm{k}\right) /\left(\mathrm{h}^{*} \mathrm{c}\right)=\left(\mathrm{h}^{*} \mathrm{c}\right) /\left(\mathrm{h}^{*} \mathrm{c}\right)=1 ; \because \mathrm{Q}^{*} \mathrm{c}^{*} \mathrm{k}=\right.$ h\}. Thus,

\section{"Unification of Gravity at only TeV Range is Possible".}

\subsection{Middle Fold [6]}

\subsubsection{Three Bottom Parts of Middle Lower Fold}

There are 3 sub-folds of Middle Lower Fold (Refer Figure 9) which are above Bottom Fold where each sub-fold includes one Type of Massive Charged Leptons viz. Tau, Muon \& Electron with their respective Massive Charge-Less Anti-Neutrinos (Right Handed) and Electro-Magnetic force carrying particles i.e. Photons. As Massive particles are defined by Straight Imaginary Line, it means imaginary curved lines in 3-D real space are Massless particles or Energy particles like, Gluons and Photons. It also shows that, these leptons should carry only Rest Mass because they are Straight lines and they gain their kinetic energy

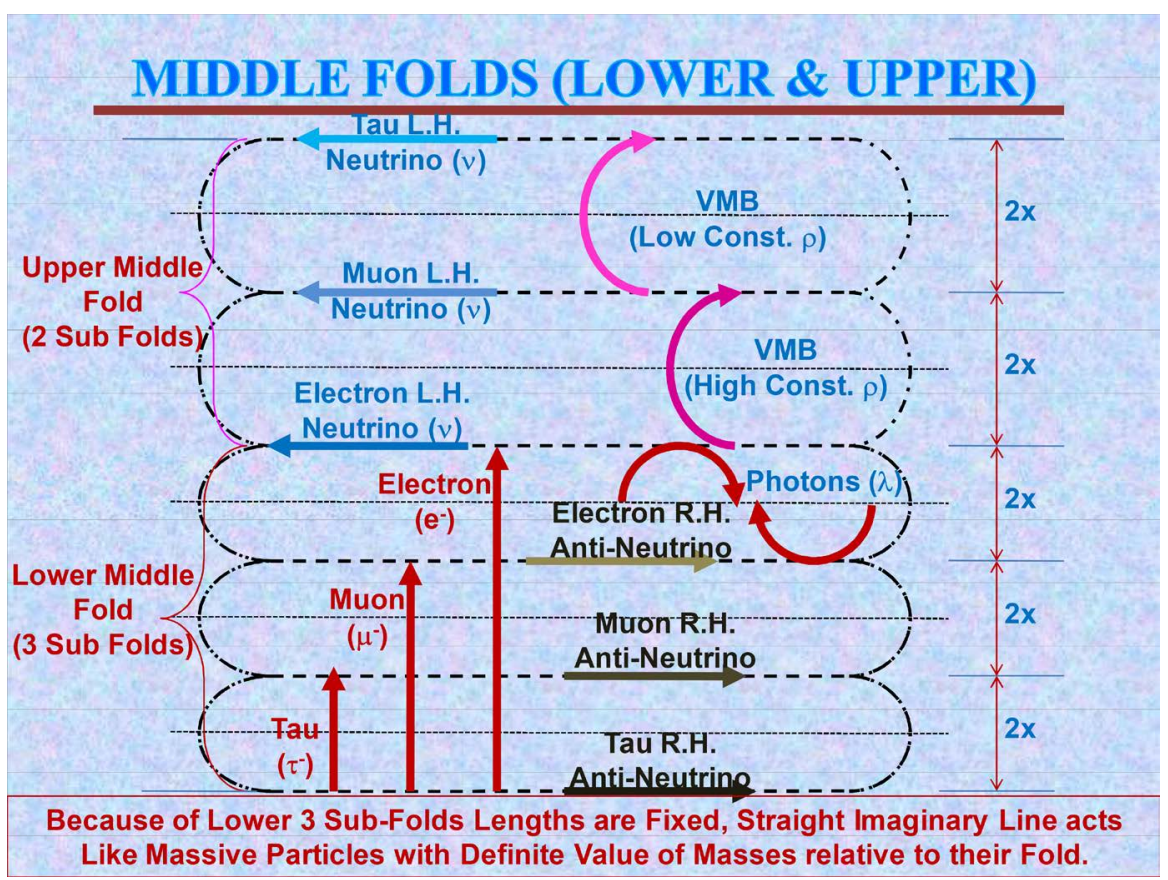

Figure 9. Middle fold. 
because of Massless photons. Thus, every moving electron carries photon/ photons with it or "Electron does not have its own kinetic energy, but owns only Rest Energy" as shown in Figure 10. Kinetic energy or motion to these electrons is given by Massless Photons which carries only Kinetic Energy (K.E. $\propto v$ ). Repulsive force between like charged particle is because of repulsive force (pressure) exerted by photons connected with these charged particles and vice a versa for attractive forces between them (For e.g. between positron and electron). Neutrinos are Charge Less because they travel horizontally in their respective 3-D Real Space.

Mass of Charge Leptons is fixed here, by means of their existence in their respective fold only. i.e., in this theory, we can calculate Mass of Particles like electron, Muon and Tau. (God doesn't play dice at least with Mass of Charged Leptons).

Mass of Charged Leptons always remains Constant like "Peas in Pod, because of Fixed Quanta Height of Each Sub-Fold."

Here, Fermions are defined as one String like Particle with one directional arrow $($ Spin $=1 / 2)$ while Bosons are made up of curved lines having rotational direction and are called as Horizontal Massless Boson Particles as they travel in 3-D Real Space.

- In case of Spin = 1 type Horizontal Massless Boson particles (Gluons, Photons), they are like Half wave line (4-D Wave) and they travel in terms of waves either Left handed or Right Handed. Propagation of wave in direction of 3-D Real Space is responsible for Magnetic Field while that in vertical $4^{\text {th }}$ Imaginary Dimension is responsible for Electric Field. Kinetic Energy (E) of Photons is directly proportional to Rotational Frequency $(v)$ of its 4-D Wave.

\section{Fermions(Leptons) Vs Bosons (Photons)}

"Electron itself does not carry any "Photons are (Massless) Kinetic Energy Kinetic Energy. It only has Rest Energy." Particles and provide Motion to Electrons.

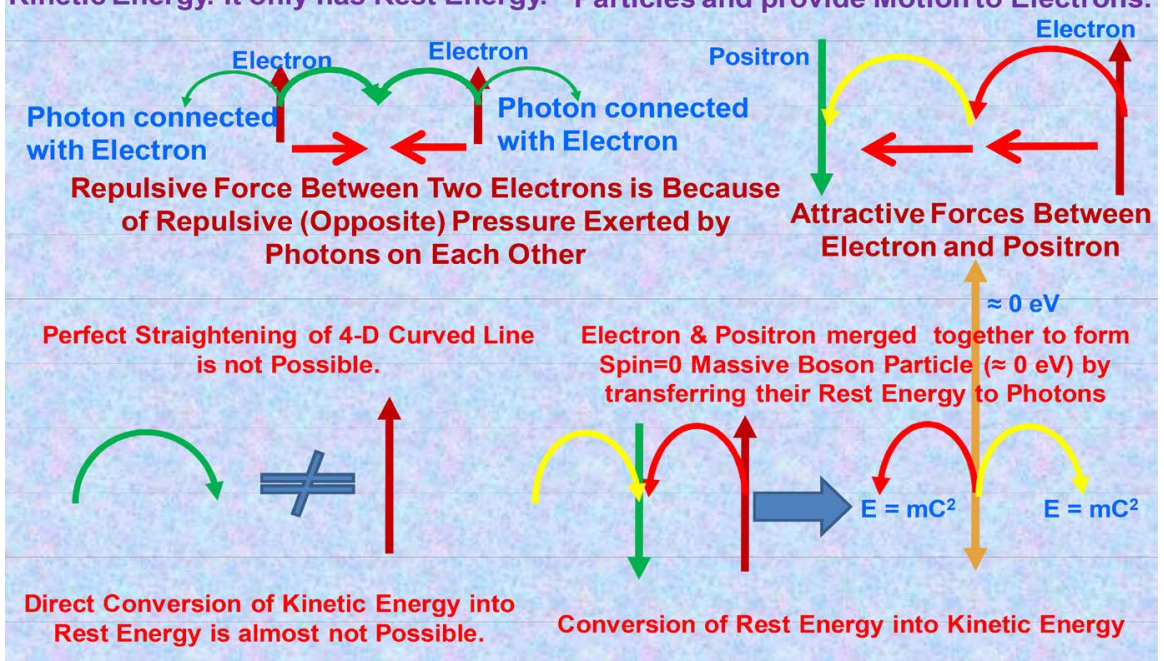

Figure 10. Horizontal massless bosons i.e. photons (kinetic energy) of massive charged fermions (rest energy). 
- For Spin $=0$ type Horizontal Massless Boson particles, they are made up of 2 Half waves of opposite spins $( \pm 1$ or \pm 2$)$ and look like Circular wave line (4-D Wave). They have either Clockwise or Anti-clockwise rotation; hence they have rotational Asymmetry (Refer Figure 11).

\subsubsection{Two Top Parts of Middle Fold}

It includes 2 sub folds for 3 Weakly Interacting Massive Neutrinos (Left Handed) and 2 types of Vertical Massless Boson Particles, VMB (Refer Figure 9). In their respective sub-fold, the energy density of all Boson particles remains same (Constant) and hence, they are called as "Equivalent to Dark Energy". It is achieved by means of, Division of Higher Constant Energy Density VMB Particle into Multiple numbers of Lower Constant Energy Density VMB Particles, when it shifts from lower sub-fold of Middle Fold to upper sub-fold in such a way that every Lower Constant Energy Density VMB Particle looks similar to each other in upper sub-fold and this feature of these VMB particles is called as "Copy Cat" future (Refer to Figure 11). Out of these two sub-folds, lower sub-fold has higher constant energy density as compare to upper sub-fold. This is similar like Atmospheric Layers over the Surface of the Earth where, air pressure decreases with increase in altitude. There are 3 Types of "VMB" particles (2 from Top parts of Middle Fold and 1 from Top Fold), such that "Constant Energy Density" of these particles decreases as they shift from lower part of Middle Fold to Top Fold. Shift in their position occurs with step by step unfolding of expanding Universe. These particles move (shift) from one sub-fold to other sub-fold, when they have spin $= \pm 1$ while they multiply each other with same constant energy density in same sub-fold, when they have spin $=0$.

\section{Horizontal Bosons (Gluon, Photon) Vs Vertical Boson (Dark Energy)}

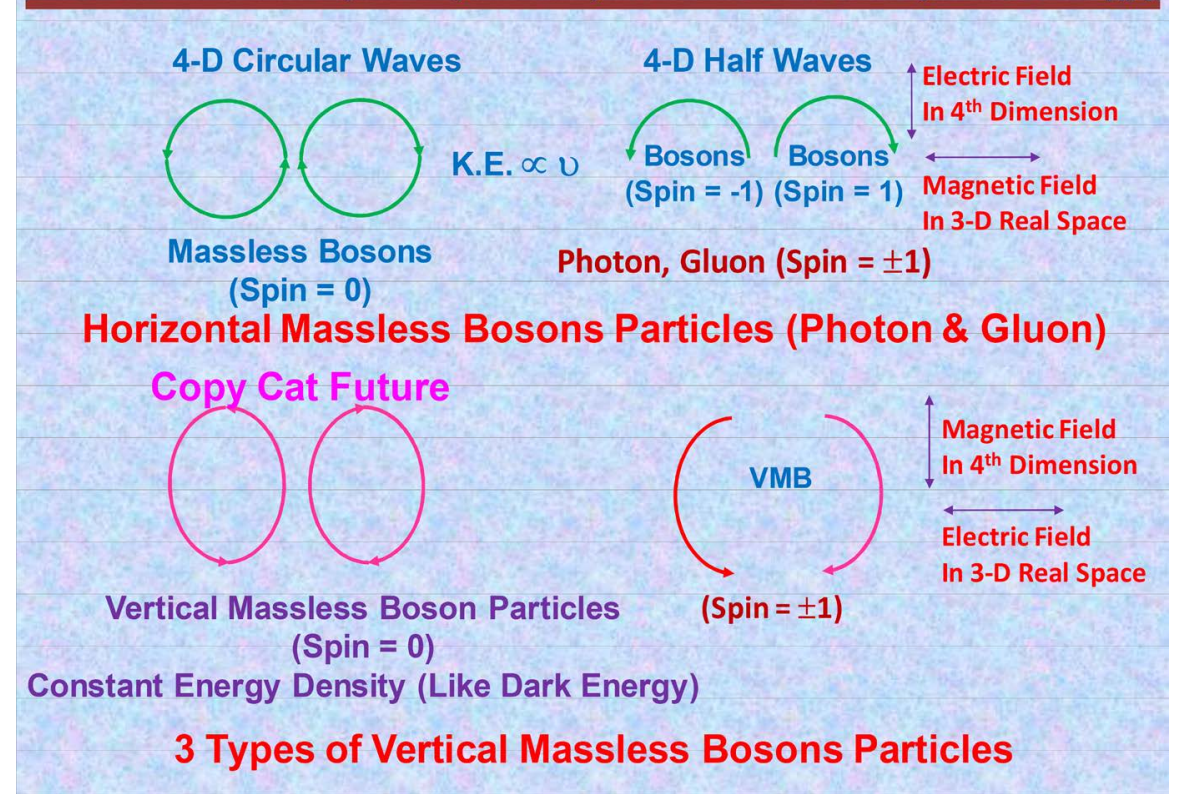

Figure 11. Horizontal massless bosons particles vs vertical massless bosons particles. 
Because of random shift in their position (as per availability of vacuum at their upper or lower sub-folds), neutrinos along with them also oscillates from one fold to other sub-fold i.e. change in type of neutrinos occurs; called as Neutrino Oscillations.

As per in Figure 12, Inner 3 Generations of Neutrino - Antineutrino come into existence by uplift of Massive Trident Particle from Bottom fold into Middle Folds and then by splitting of its each axis through Diagonal Separation, it gives Massiveness as well as Left Handedness properties to Neutrinos. Standard Model can't explain these properties of Neutrinos because of non-prediction of Trident particle. It means discovery of Trident particle is very necessary to prove Left handedness and Massiveness of Neutrinos.

Note that, this alternate source of energy (VMB particles) has property of Negative pressure (Constant Energy Density) and hence, they are Higher $4^{\text {th }}$ Dimensional Anti-Gravity Energy. It is just above Middle Lower Fold of Electron. If it becomes possible to harness this pure form of energy, miraculous changes in aviation and many more scientific fields are expected.

\subsection{Top Fold}

As shown in Figure 13, from Bottom Fold creates Top Fold through creation of Higgs Boson particle $($ Spin $=0$ ) by internal rotation of one of the Massive axis of Trident particle. Along with Higgs Boson, Higgs Field (VMB Particle at L.H. side of Bottom Fold) also transferred into Top Fold. Top of Top Fold consists 4th type of L.H. Neutrino which yet to be discovered. This Theory demands existence of 4th pair of Neutrino and Anti-neutrino; discovery of them will act as solid proof of this theory.

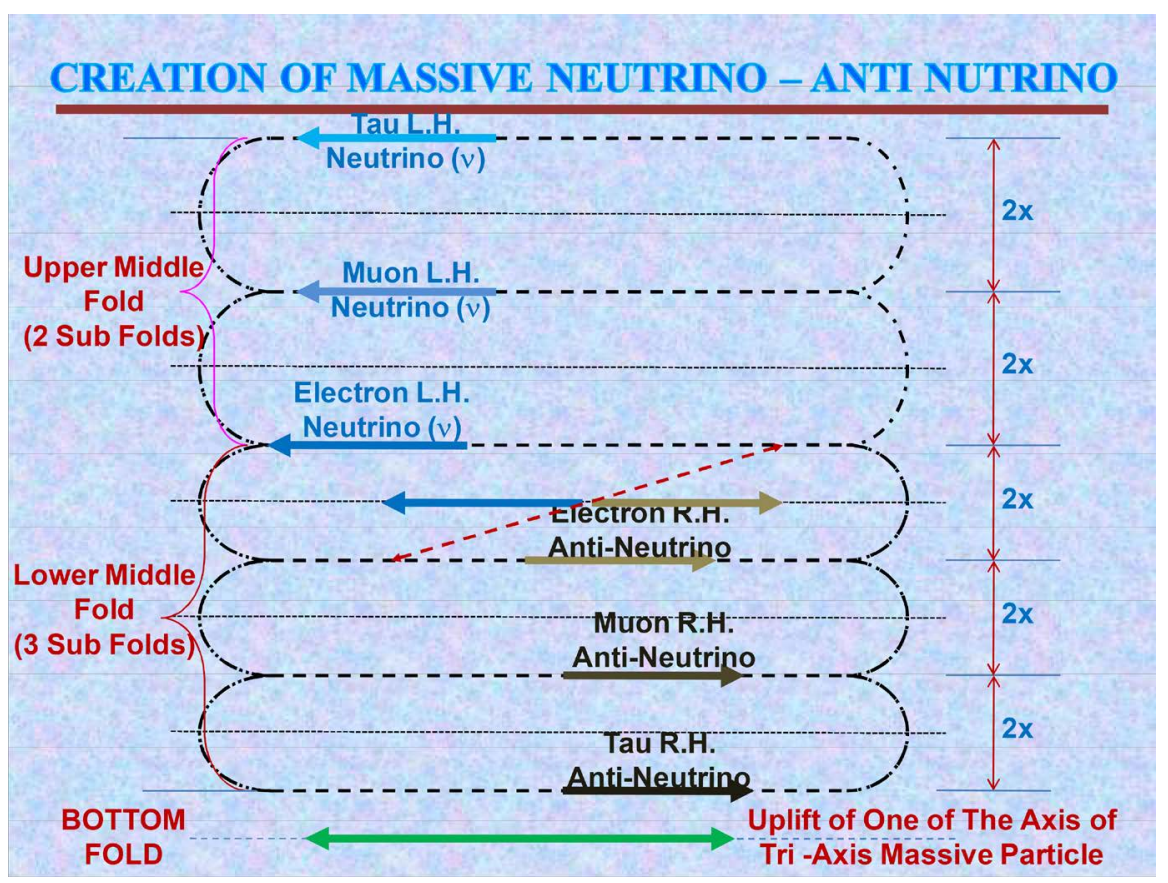

Figure 12. Creation of pair of massive neutrinos and antineutrinos. 


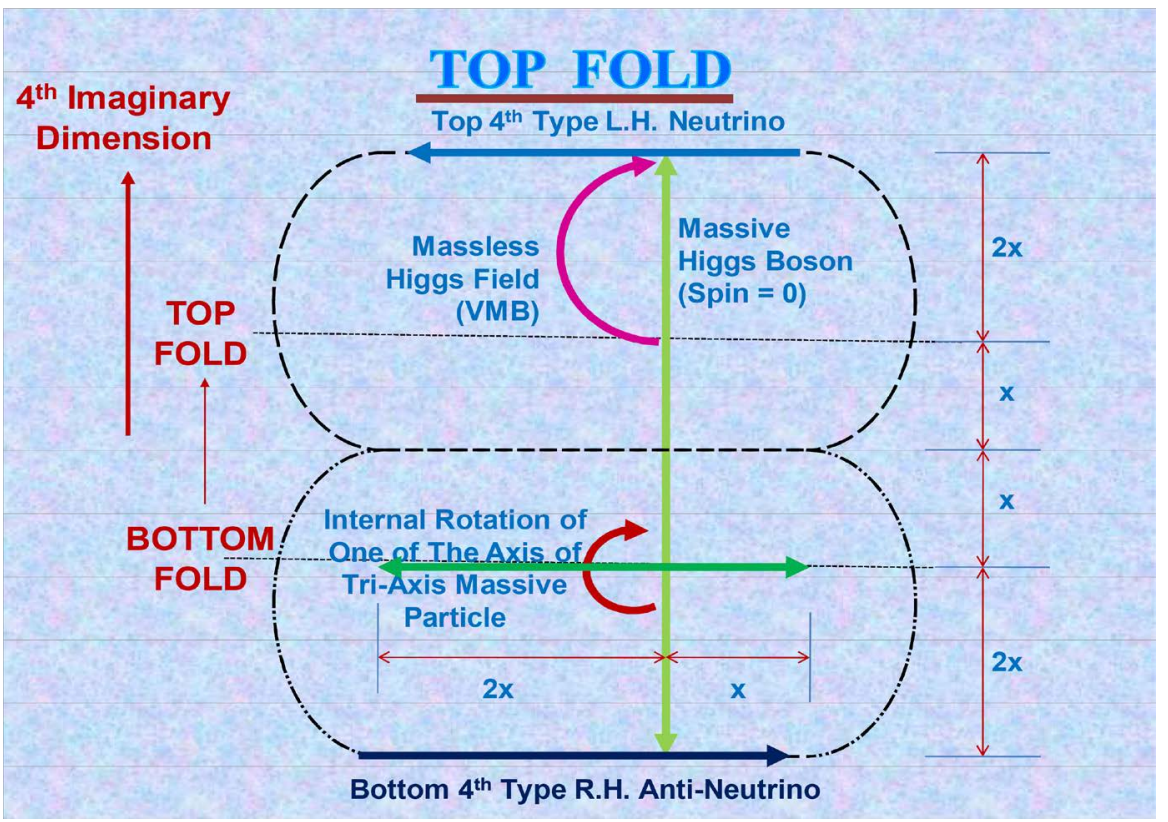

Figure 13. Creation of top fold.

4th Dimension is Imaginary Dimension and as per equivalence of Mass and Imaginary Line, vertical height of this dimension represents Mass of Particle. Thus, through vertical upward motion, Massive Higgs Boson particle creates Middle Folds and indirectly gives mass to Charged Lepton particles through creation of Middle Lower Folds.

\section{Creation of Bottom Fold in 4 Dimensional Universe [6]}

Rotational Symmetry of Massless Boson (Spin $=0)$ particle as shown in Figure 14, was spontaneously broken by diagonally downward shift of R.H. Massive Boson Particle (TeV).

Downward pressure of Two Massless Boson particles caused Left end of R.H. Massive Boson Particle to shift diagonally in downward direction. It is equivalent to (Anti clockwise) rotational shift. This Diagonal Massive Boson (Spin =0) particle converted into Quadra Particle i.e. into 4 type of particles. One Pair is made of Vertical (4 ${ }^{\text {th }}$ Dimensional) Massive Boson particles with opposite charges $( \pm Q)$ on each particle (instead of $2 \mathrm{Q} / 3$ ) and second Pair is made up of Horizontal (3 Dimensional) Massive Boson particles. Out of $1^{\text {st }}$ Pair, Charge on R.H. Upward directional particle is $+\mathrm{Q}$ and it is called as $4^{\text {th }}$ Quark; while charge on L.H. Downward directional particle is -Q and it is called as $4^{\text {th }}$ Anti-Quark. In $2^{\text {nd }}$ Pair, Left Handed topmost particle is charge less (Charge $\left.=0\right)$ Massive Boson particle and is called as $4^{\text {th }}$ Top Neutrino $\left(v_{\mathrm{T}}\right)$; while Right Handed Bottommost particle is also charge less (Charge $=0$ ) Massive Boson particle and is called as $4^{\text {th }}$ Bottom Anti-Neutrino $\left(v_{\mathrm{B}}^{\prime}\right)$. This Pair of Top Neutrino and Bottom Anti-Neutrino is $4^{\text {th }}$ Pair other than 3 Pairs of Neutrino and Anti-Neutrino as per Standard Model of Particle Physics. Creation of $4^{\text {th }}$ pair of Neutrinos from R.H. Massive Boson particle gives Massiveness and Left handedness properties to 


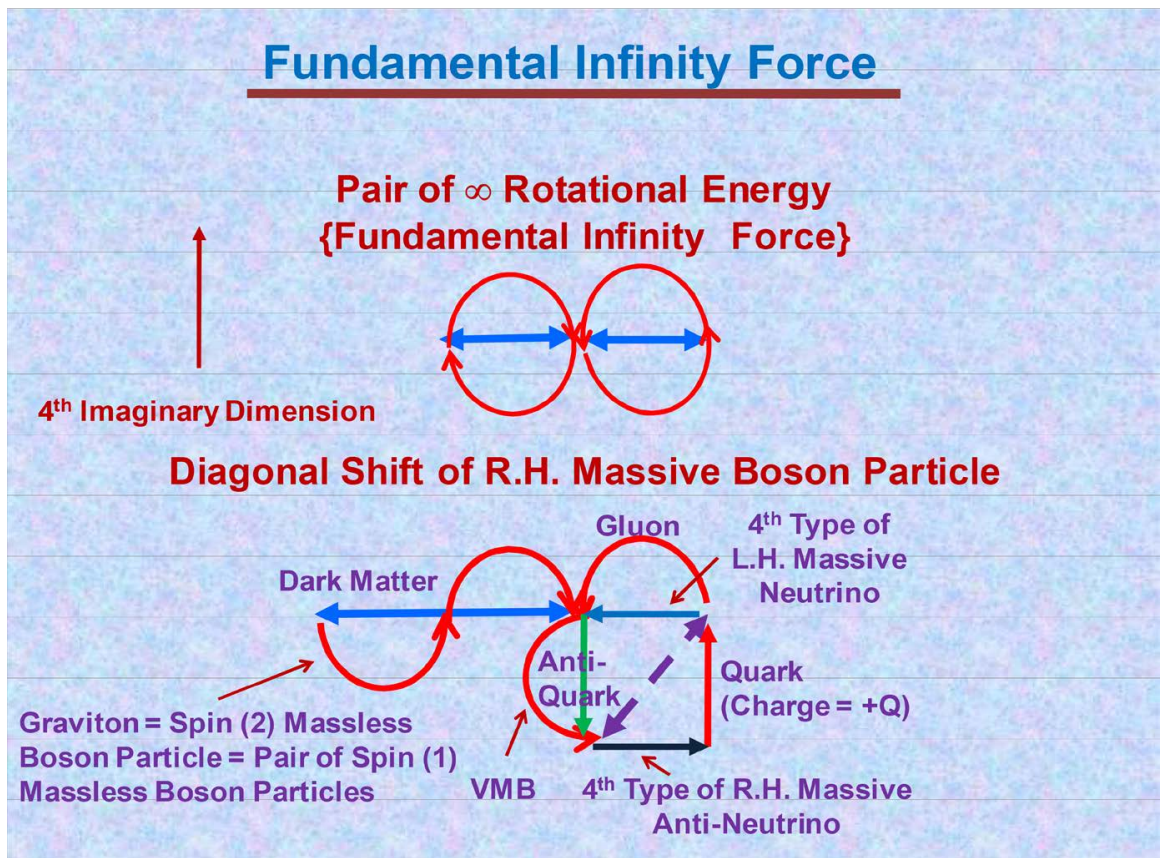

Figure 14. Infinity $(\infty)$ like rotation of massless boson $(\mathrm{Spin}=0)$ particles at $\mathrm{TeV}$ range.

Neutrinos and Mass to Quarks.

Apart from this Quadra Massive Particle, you will see creation of Graviton (Massless Boson, Spin $=2$ ) particle on Left hand side of Figure 14. Though graviton moves forward in space with speed of light, c, but still it has direction opposite to its forward propagation velocity, that means towards Quadra Particle or Center of Quarks (which later converts into Hadrons i.e. materialistic world). This is the main reason behind attractive force of Gravity. Increase in Curvature of Space Time is equivalent to Decrease in Wavelength i.e. Increase in Kinetic Energy of Graviton. On Nuclear Level, Gravity Force acts on Matter (Quarks) from one Side i.e. Left Side only. This Graviton is guided by Massive Boson $($ Spin $=0)$ Particle called as Dark Matter. With the help of Gravitons, Dark Matter gravitates. Nature of Dark Matter is to expand in Space-Time while that of Graviton is to contract/ Compress or gravitate it.

Further creation of Bottom Fold in terms of Hadrons i.e. Pair of 3 Quarks is shown below in Figure 15. From this Figure, it is seen that, during creation of the Universe, there were equal amount of Quarks (R.H. Side) and Anti-Quarks (L.H. Side), but later on anti-quarks convert into quarks through internal rotation of L.H. VMB as discussed earlier (Refer to Figure 7).

In following Figure 16, arrangement of all Fundamental particles in their respective Folds in $4^{\text {th }}$ Imaginary Dimension and true nature of all these particles are explained through "One Simple Diagram".

Some interesting and Empirical formulas of this theory are mentioned below, which may help to find relation between Fundamental Constant and Natural Constants like Mass of Electron, Neutron etc.:

- $\mathrm{Mc}=$ Critical Mass of the Universe $=\left(\mathrm{H}_{\mathrm{Q}}\right)^{2}=\left\{\mathrm{c} /\left(2^{\star} \mathrm{Pi}^{\star} \mathrm{Q}\right)\right\}^{2} \approx 8.9 \times 10^{52} \mathrm{Kg}$ 

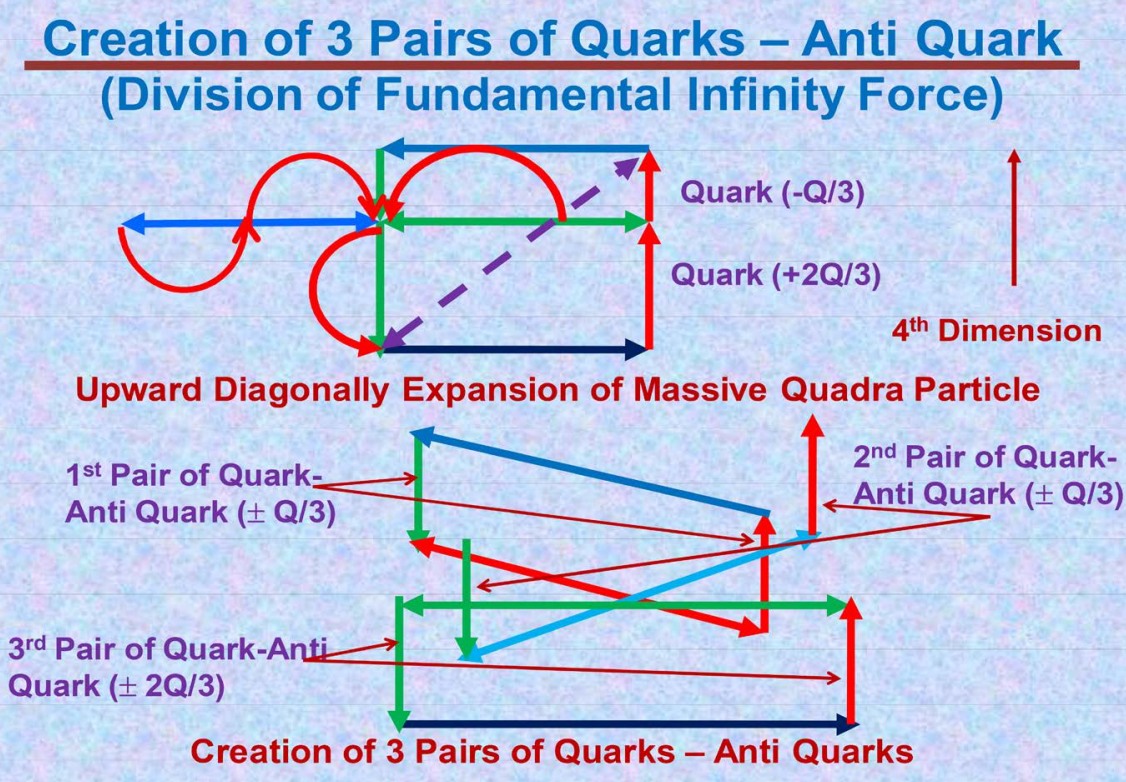

Figure 15. Creation of 3 pairs of quarks-anti quarks and trident particle in bottom fold.

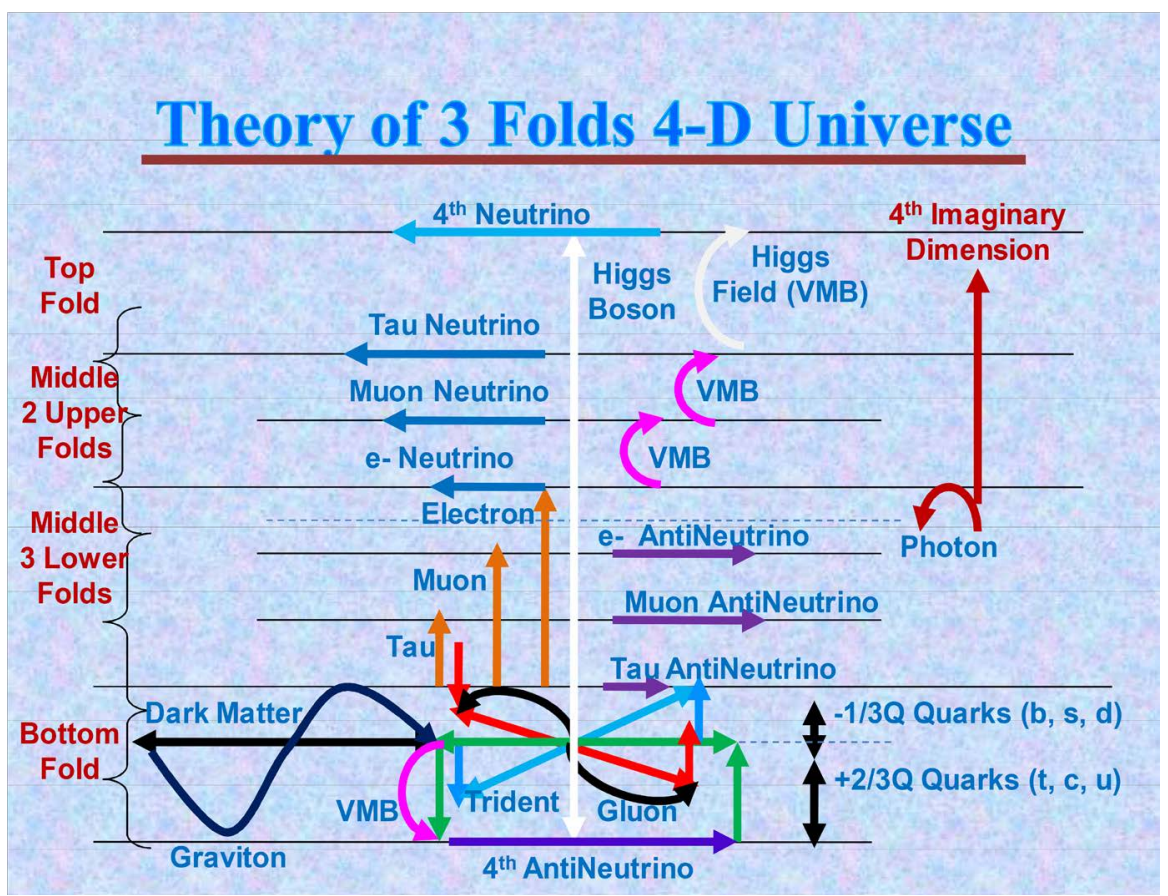

Figure 16. Arrangement of all fundamental particles in their respective folds.

- Initial Size of Universe in 3-D Space $=\left(\mathrm{R}_{\mathrm{U}}\right)_{\text {Initial }}=\left\{\mathrm{M}_{\mathrm{c}}{ }^{\star} 2 \pi / \mathrm{k}\right\}^{1 / 3 *} \mathrm{Q} \approx 5.5 \times 10^{6}$ $\mathrm{m}$ (Closer to Radius of the Earth)

- $\left(4^{*} \mathrm{k} / \pi^{2}\right) \cong \pi \mathrm{TeV}$

- $\mathrm{M}_{\mathrm{e}}=$ Mass of Electron $=\mathrm{E}_{\mathrm{e}} / \mathrm{c}^{2}=\left\{\mathrm{h}^{*} \mathrm{v}_{\mathrm{e}}\right\} / \mathrm{c}^{2}=\left\{\mathrm{h}^{*}\left(2^{*} \pi^{2} / \mathrm{Q}\right)\right\} / \mathrm{c}^{2}=9.09 \times 10^{-31} \mathrm{Kg}$ $=\left\{\left(2^{\star} \pi^{2 *} \mathrm{c}^{\star} \mathrm{k}\right) / \mathrm{Q}\right\} \mathrm{eV} \approx 0.51 \mathrm{MeV} ; \because$ Freq. of Electron, $v e=\left(2^{\star} \pi^{2}\right) / \mathrm{Q}$

- $\left\{\mu_{0} /\left(4^{\star} \pi\right)\right\}=\left(k^{\star} \alpha\right) /\left(2^{\star} \pi^{\star} \mathrm{Q}\right) ; \alpha=$ Fine Structure Constant $\approx 1 / 137$

- $\mathrm{M}_{\mathrm{N}}=$ Mass of Neutron $=\pi^{\star} \mathrm{h} /\left(\mathrm{c}^{2 \star} \mathrm{k}\right)=1.673 \times 10^{-27} \mathrm{Kg}=\left(\pi^{*} \mathrm{c}\right) \mathrm{eV} \approx 941$ 
$\mathrm{MeV} ; \because$ Frequency of Neutron, $v_{\mathrm{N}}=\pi / \mathrm{k}$

\section{Conclusion}

In this theory, "imaginary straight line" is proved as equivalent to "mass of particle", while any curved imaginary line shows massless equal to Spin 1 property of Boson particles viz. Gluons and photons. Thus, massless is directly connected with spin $=1$ property of photon which is already proved in QED theory. True natures of photons and dark energy equivalent particles i.e. VMBs are revealed through their unique characteristics viz. Horizontal 3-D space motion for photons and vertical 4th dimensional motion for VMB. This vertical motion also gives one more property to VMB i.e. Constant energy density by means of copy cat future in the same sub-fold of upper middle fold. These VMB particles not only create repulsive force (Inflation) in terms of higgs field $($ Spin $=0)$ in the universe, but through vertical upward motion, they convert L.H.S. anti-quarks into quarks. Thus, they played the major role in solving the mystery of anti-matter and matter asymmetry in a very beautiful and elegant way. Also, arrangement of all particles in 3-Folds way not only simplifies particle physics theory but, also gives unification of physics and wide scope to think "beyond standard model", within $\mathrm{TeV}$ range itself. The discovery of new particles predicted by this theory like $4^{\text {th }}$ pair of neutrinos, trident particle, VMBs will solve lots of problems of standard model of particle physics. Finally, author concludes that with advancement in this theory, new era of science will begin for a better future of mankind.

\section{Acknowledgements}

I would like to thank my family members for their valuable supports given to me.

\section{Conflicts of Interest}

The author declares no conflicts of interest regarding the publication of this paper.

\section{References}

[1] Harald, F. (2009) The Fundamental Constants: A Mystery of Physics. World Scientific Publishing, Co., Singapore.

[2] Aitchison, I.J.R. and Hey, A.J.G. (2002) Gauge Theories in Particle Physics. Vol. 1, CRC Press, Boca Raton.

[3] Michele, M. (2005) A Modern Introduction to Quantum Field Theory. Oxford University Press, Oxford.

[4] David, H., Robert, R. and Jearl, W. (2013) Fundamental of Physics Extend. 10th Edition, John Wiley \& Sons, Inc., Hoboken.

[5] Malcolm, L. (2013) Quantum Concepts in Physics: An Alternative Approach to the Understanding of Quantum Mechanics. Cambridge University Press, Cambridge.

[6] Yogesh, V.C. (2018) The Creation of the Universe (Beyond the Big Bang-Unification of Physics). International Journal of Current Research, 10, 75820-75832. 